\title{
Comparison of Flow and Dispersion Properties of Free and Wall Turbulent Jets for Source Dynamics Characterisation
}

\author{
S. S. Aloysius and L. C. Wrobel \\ School of Engineering and Design, Brunel University, Uxbridge UB8 3PH, UK
}

\begin{abstract}
The objective of this paper is to provide an investigation, using large eddy simulations, into the dispersion of aircraft jets in co-flowing take-off conditions. Before carrying out such study, simple turbulent plane free and wall jet simulations are carried out to validate the computational models and to assess the impact of the presence of the solid boundary on the flow and dispersion properties.

The current study represents a step towards a better understanding of the source dynamics behind an airplane jet engine during the take-off and landing phases. The information provided from these simulations can be used for future improvements of existing dispersion models.
\end{abstract}

\section{Software availability}

Software name: FLUENT 6.3

Developers: FLUENT Inc

Contact address: 10 Cavendish Court, Lebanon, NH 03766, USA. info@fluent.com.

Software requirements (for our cluster simulations): Linux X86-64 Opensuse. Tecplot used for post-processing.

Hardware requirements (for our cluster simulations): 8 dual core Intel Pentium $43.0 \mathrm{GHz}$ with shared type memory.

Program size: $280-330 \mathrm{MB}$

Availability and cost: Contact FLUENT Inc or use website http://www.fluent.com.

\section{Introduction}

Air transportation accounts for 3.5\% of global warming from human activity, and this figure is predicted to rise up to $15 \%$ by 2050 if no limits are imposed, according to the international panel on climate change (Penner et al., 1999). Global effects such as global warming and the depletion of the ozone layer are also directly affected by air transport activities.

The aviation industry has been subjected to ever-increasing pressure to reduce its environmental footprint. For this to be achieved, airfield regulators and operators rely on dispersion modelling software, based on Lagrangian or Gaussian techniques, to quantify the current situation and to make accurate predictions of future scenarios.

Thrust for a jet aircraft is provided by its engines through hot gases ejected from the exit nozzle. Combustion is a necessary process for the engine to work. In an ideal situation, the combustion of aircraft fuel will only produce carbon dioxide $\left(\mathrm{CO}_{2}\right)$ and water $\left(\mathrm{H}_{2} \mathrm{O}\right)$ (Penner et al., 1999). In real conditions, residual products such as soot, hydrocarbons (HC) and carbon monoxide (CO) will form from "non-ideal combustion processes" and from the oxidation of nitrogen contained in air, the socalled nitrogen oxides $\left(\mathrm{NO}_{\mathrm{x}}\right)$ (Archer and Saalas, 1996). 
NOx emissions from aircraft sources around Zurich airport in the year 2003 were found to be approximately $80.7 \%$ of the total emissions (Celikel et al., 2004). Unfortunately, these emissions are not properly accounted for in most commonly used dispersion modelling software because of the spatial and time resolutions needed for a complete airfield simulation, and an increased knowledge of local source dynamics is needed for their proper inclusion into those models.

Recent studies have seen the introduction of an advanced Eulerian CFD technique based on Large Eddy Simulation (LES) for studying local emission dispersion at an airport (Aloysius et al., 2006). It was found that this model is too computationally demanding for airport management use at the present but, on the other hand, it can provide a better insight on the fluid mechanics and valuable source dynamics information for Gaussian and Lagrangian models.

Hence, the objective of the present study is to simulate numerically the dispersion of aircraft jets in co-flowing take-off conditions. The CFD formulations will be first validated with available theoretical and experimental results for simple free and wall jets in a nearly two-dimensional non-buoyant condition, before including buoyancy effects in a complete three-dimensional simulation. This is the first step of a staged approach aimed at increasing the understanding of the near-field effects of aircraft exhaust plumes under realistic conditions.

\section{Numerical methods}

The CFD solver FLUENT 6.3 is used in the present study. FLUENT is a commercial CFD package with the capability to complete high accuracy simulations based on a number of numerical methods.

This study employs LES to solve the general Navier-Stokes equations. The basic concept of a LES model is to explicitly solve the larger eddies of the control volume which contain most of the energy, do most of the transport of conservation properties, and are dependent on the flow geometry and boundary conditions, whereas the smaller eddies are modelled through a filtering process due to their universality and their lesser influence on the fluid flow (Bakker, 2004). The filtered Navier-Stokes equations in terms of the subgrid-scale Reynolds stresses can be written as (Fluent, 2005):

$$
\frac{\partial \bar{u}_{i}}{\partial t}+\frac{\partial \overline{u_{i} u_{j}}}{\partial x_{j}}=-\frac{\partial \bar{P}}{\partial x_{i}}+v \nabla^{2} \overline{u_{i}}-\frac{\partial \tau_{i j}}{\partial x_{j}}
$$

with the subgrid-scale Reynolds stresses $\tau_{i j}=\overline{u_{i} u_{j}}-\overline{u_{i}} \overline{u_{j}}$.

The conventional Smagorinsky subgrid-scale model hypothesis is that the subgrid-scale Reynolds stresses $\tau_{i j}$ are also proportional to the rate of strain tensor:

$$
\tau_{i j}=-2 \mu_{t} \overline{S_{i j}}
$$

with $\overline{S_{i j}}=\frac{1}{2}\left(\frac{\partial \overline{u_{i}}}{\partial x_{j}}+\frac{\partial \overline{u_{j}}}{\partial x_{i}}\right)$ the rate of strain tensor for the resolved scale and $\mu_{t}$ the subgrid scale turbulent viscosity. This model gives a relationship between the subgrid scale turbulent viscosity and the rate of strain tensor in the form: 
with $|\bar{S}|$ the filtered rate of strain defined by $|\bar{S}|=\sqrt{2 \overline{S_{i j} \overline{S_{i j}}}}, L_{s}$ the Smagorinsky length scale which is proportional to the filter width $\Delta$ and the Smagorinsky constant $C_{s}$. In this study, the Smagorinsky constant $C_{s}$ is determined dynamically as a function of time and position through another type of filtering process called a test filter (Fluent, 2005).

The transport process of the species is determined through the calculation of the mass diffusion equation (Fluent, 2005):

$J=\left(\rho D+\frac{\mu_{t}}{S c t}\right) \nabla Y$

where $J$ is the diffusion flux, $D$ the diffusion coefficient of the species, $Y$ the mass fraction of the species, $\mu_{t}$ the turbulent viscosity, $\rho$ the density of the species and Sct is the turbulent Schmidt number, equal to $\frac{\mu_{t}}{\rho D_{t}}$.

The pressure-velocity coupling uses the Consistent Semi Implicit Method for the Pressure Linked Equation (SIMPLEC) to derive the pressure from the continuity equation through an iterative process of correcting time steps after an initial guess (Fluent, 2005). The discretization scheme used for the control volume is the secondorder accurate bounded central difference scheme for the momentum, energy and species transport equation (Fluent, 2005).

\section{Results and discussions}

The study of horizontal turbulent jets has been an important topic for many years. The earliest experimental investigation was conducted by Forthmann (1934) on a plane turbulent jet (Rajaratnam, 1976). Since then, free jets have been studied with different experimental equipments such as pitot tubes (Forthmann, 1934), Hot Wire Anemometry (Everitt and Robins, 1978), Laser Doppler Anemometry (Ramaprian and Chandrasekhara, 1985) and Particle Image Velocimetry (Mi et al., 2007).

The initial part of this section will present validation studies using plane free and wall jets. According to Ribeiro and Whitelaw (1980), plane jets were more thoroughly investigated than round jets due to their slower rate of mean velocity decay. Rodi (1975) provided a description of two-dimensional behavioural commonalities between the two jets. A plane jet issuing from a rectangular nozzle with infinite width and finite height can be considered as 2D, as can an ideal round jet due to its axi-symmetry, but the similarities stop here. Haworth and Pope (1987) reported some differences between the two flows especially in terms of spreading rate. For the plane jet, they used Rodi's (1975) spreading value of 0.11 for a comparison with their simulation results. This value was also reported by Everitt and Robins (1978) in their experimental studies of jets in still and moving streams. Other researchers reported spreading rates varying from 0.087 to 0.128 (Haworth and Pope, 1987). For a round jet, Haworth and Pope (1987) agreed with the value of 0.086 reported by Wygnanski and Fiedler (1969) and Rodi (1975). Mi et al. (2005) showed that, for the plane jet, the centreline decay of the mean velocity is proportional to the 
streamwise distance to the power minus one-half $\left(x^{-1 / 2}\right)$, while the half-width varies linearly with the streamwise distance in a region not far downstream from the potential core. For the round jet, on the other hand, the centreline decay of the mean velocity is proportional to the inverse of the streamwise distance $\left(x^{-1}\right)$. Mi et al. (2000), in another study comparing different types of nozzles, found that the average length of the potential core is higher for circular jets that those issuing from noncircular geometries. This statement implies that there is more entrainment with the surroundings for any non-circular jet than for a round jet (Mi et al., 2000). Closer to the jet exhaust, the rate of decay is lower for the round jet than its non-circular counterparts. Deo et al. (2007) observed that round jets "produce weaker velocity fluctuations" when compared to plane jets, and reported that this difference is a proof of the "influence of nozzle exit geometry on the exit velocity fields" of the plane and round jets (Deo et al., 2007).

\subsection{Validation}

Validation of the CFD formulation was carried out for the simple cases of a plane jet in an unbounded and in a partially bounded environment, to test the grid setting of the control volume and verify the accuracy of the model used. Forthmann's experimental setup was utilised for these simulations (Forthmann, 1936); a twodimensional control volume was created to replicate the experiment (Fig. 1). Forthmann's experiments were chosen for the validation studies as he reported detailed results for both free and wall jets. The amount of data available is substantial as many researchers have compared their results to Forthmann's for the characterisation of self-similarity profiles. The simulation results are also compared with analytical results of Tollmien (Abramovich, 1963) and Goertler (Abramovich, 1963), Zijnen's Gaussian profile (Van der Hegge Zijnen, 1958), a sech ${ }^{2}$ profile (Thorne, 2004), and recent analytical expressions of Morchain et al. (2000) and Aziz et al. (2008).

Both Abramovich (1963) and Rajaratnam (1976) provided details of the boundary conditions for the simulations. The inlet jet velocity is assumed to be $35 \mathrm{~m} / \mathrm{s}$ issuing from a slot with aspect ratio of 21.7 and height $b_{0}=0.03 \mathrm{~m}$, which gives a Reynolds number of 105,000 . The sides adjacent to the jet were set up as walls.

\subsubsection{Free Jet}

Initial tests performed to define the extent of the computational domain suggested that a control volume of dimensions $146.6 b_{0}$ by $106.6 b_{0}$, where $b_{0}$ is the jet height, is sufficiently large to avoid influences from the artificial boundaries. A mesh sensitivity analysis was then conducted for that particular control volume. This comprised three tests with different mesh densities, by varying the number of grid points and the grid spacing on different edges numbered 1, 2 and 3 in Fig. 2 and Table 1. These variations take into account that, according to Forthmann's results, the jet core has a Bell Shape (BS) distribution profile. The BS scheme in Fluent distributes the edge mesh density as a normal distribution; when $\mathrm{BS}<0.5$, the mesh is densest near the edge centre and less dense near the edge extremities (Fluent, 2005). The other edges have a simple Successive Ratio (SR) grading, where the length of the following edge is multiplied by a constant ratio. The final mesh density for the considered control volume consists of 57,500 cells and 57,981 nodes. 
As can be seen in Fig. 3, the results of the CFD simulation closely follow the analytical results, all of which provide similar curves; in particular, good agreement is obtained with the Goertler profile in the inner part of the flow and the Tollmien curve in the outer part of the flow.

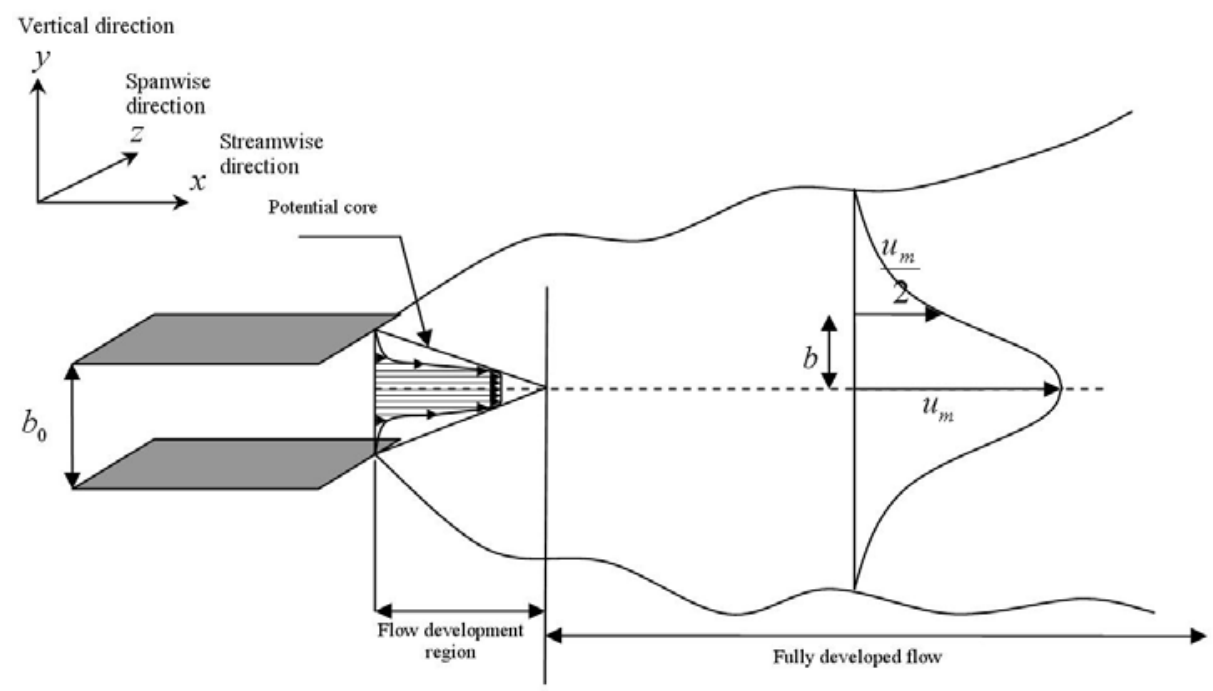

Plane free jet

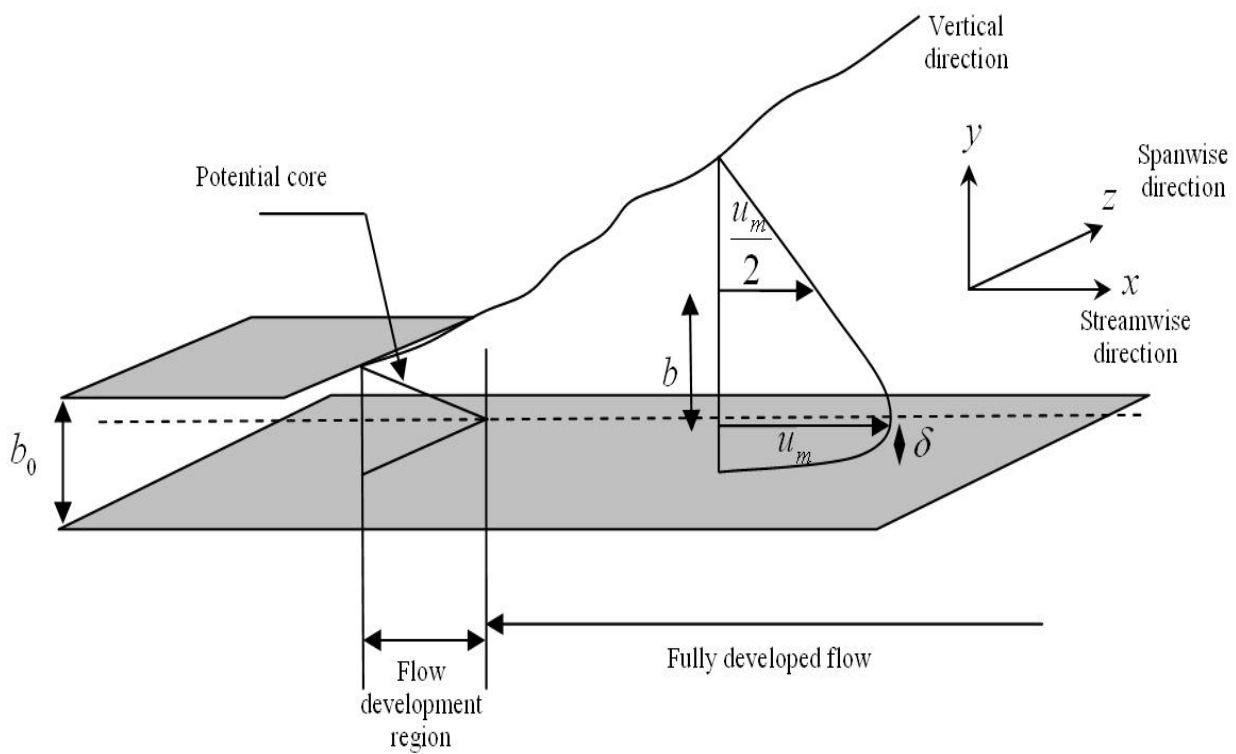

Plane wall jet

Fig. 1 - Geometry of Forthmann's experimental setup 


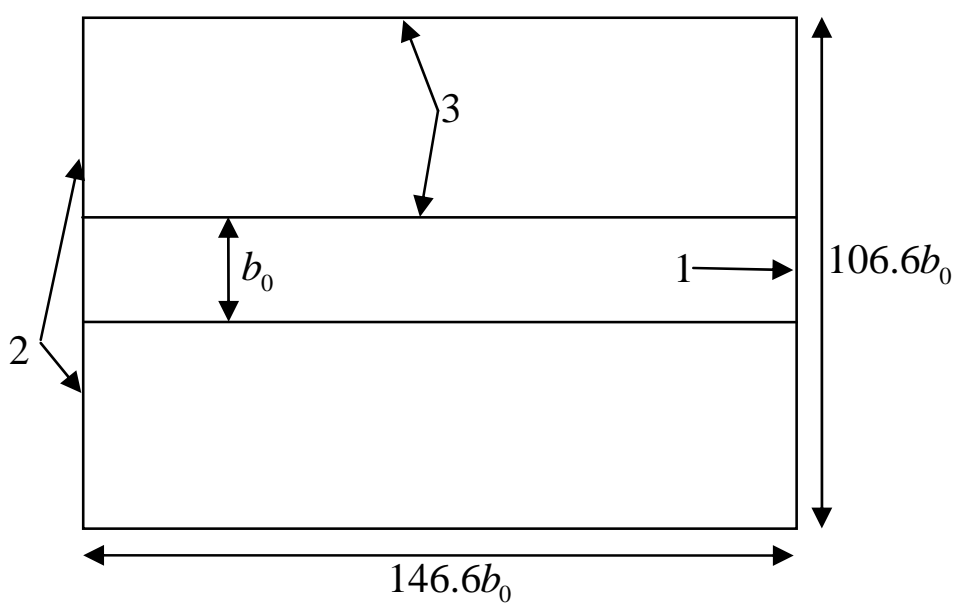

Fig. 2 - Geometry setup of the control volume

\begin{tabular}{|c|c|c|c|c|}
\hline Tests & 1 & 2 & 3 & $\begin{array}{c}\text { Mesh } \\
\text { Density }\end{array}$ \\
\hline$\# 1$ & $\begin{array}{c}15 \\
\mathrm{BS}=0.45\end{array}$ & $\begin{array}{c}50 \\
S R=1.04\end{array}$ & $\begin{array}{c}125 \\
\mathrm{SR}=1.02\end{array}$ & $\begin{array}{c}14,375 \\
\text { cells }\end{array}$ \\
\hline \#2 & $\begin{array}{c}25 \\
\mathrm{BS}=0.45\end{array}$ & $\begin{array}{c}75 \\
S R=1.04\end{array}$ & $\begin{array}{c}200 \\
S R=1.02\end{array}$ & $\begin{array}{c}35,000 \\
\text { cells }\end{array}$ \\
\hline \#3 & $\begin{array}{c}30 \\
\mathrm{BS}=0.45\end{array}$ & $\begin{array}{c}100 \\
S R=1.04\end{array}$ & $\begin{array}{c}250 \\
S R=1.02\end{array}$ & $\begin{array}{c}57,500 \\
\text { cells }\end{array}$ \\
\hline
\end{tabular}

Table 1 - Mesh distribution and tests performed

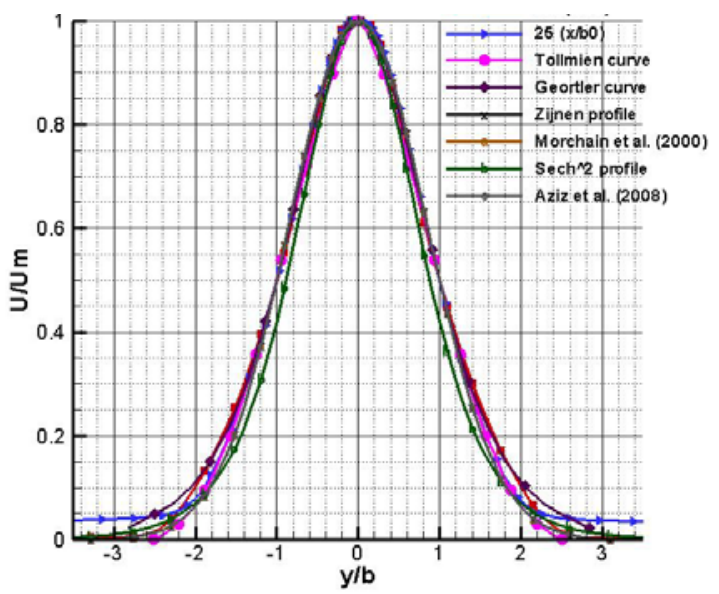

Fig. 3 - Vertical velocity comparison between CFD and theoretical methods 
Wygnanski and Fiedler (1969) reported that the changes in velocities and velocity fluctuations are more pronounced in the vertical direction than in the streamwise direction, with a substantial scatter at the edges of the flow. Everitt and Robins (1978) also highlighted this problem and reported that this was the major cause of experimental errors encountered using HWA, because of the "high ratio of the velocity fluctuations" present in the outer region of the flow. Ramaprian and Chandrasekhara (1985) also emphasised this point and concluded that this has a consequence in generating errors "due to nonlinearity and large changes in instantaneous flow orientation”. According to List (1982) and later confirmed by Namer and Otugen (1988), results found with HWA "underestimate turbulence intensity and overestimate the mean velocity near the edges of the jet".

Kotsovinos (1976) found that, instead of using the distance where the velocity reaches zero, the distance where the velocity attained half the axial velocity $(b)$ is of greater value. He also reported that the spreading growth of the jet can be considered as linear, but this is only valid up to 200 diameters behind the jet. Unlike other researchers, Kotsovinos (1976) provided a simple but efficient mathematical representation of the rate of spread that can be compared directly with the CFD data. Moreover, he provided valuable data related to Forthmann's experimental results, necessary for the following comparison. Kotsovinos (1976) concluded that all the experimental work he analysed would fit a single curve with the equation:

$$
\frac{b}{b_{0}}=K_{1}\left(\frac{x}{b_{0}}+K_{2}\right)
$$

where $K_{1}$ and $K_{2}$ are coefficients derived from experiments. From Forthmann's experiment, Kotsovinos established the values $K_{1}=0.096$ and $K_{2}=0.6$ (Kotsovinos, 1976).

By correlating each experiment, Kotsovinos derived a unique third-order polynomial equation (Kotsovinos, 1976):

$$
\frac{b}{b_{0}}=0.228+0.0913\left(\frac{x}{b_{0}}\right)+0.00005101\left(\frac{x}{b_{0}}\right)^{2}+0.000000331\left(\frac{x}{b_{0}}\right)^{3}
$$

which is only valid up to 200 diameters behind the jet. Fig. 4 presents a correlation between Kotsovinos's curve with Forthmann's and CFD plots.

All three curves are in very good agreement. As expected, Forthmann's and Kotsovinos' results are the closest because Kotsovinos used Forthmann's data to derive his equation. The slight difference concerning the CFD solution might have come from the way the parameter $b$ was found, since $b$ was interpolated from the velocity profile. 


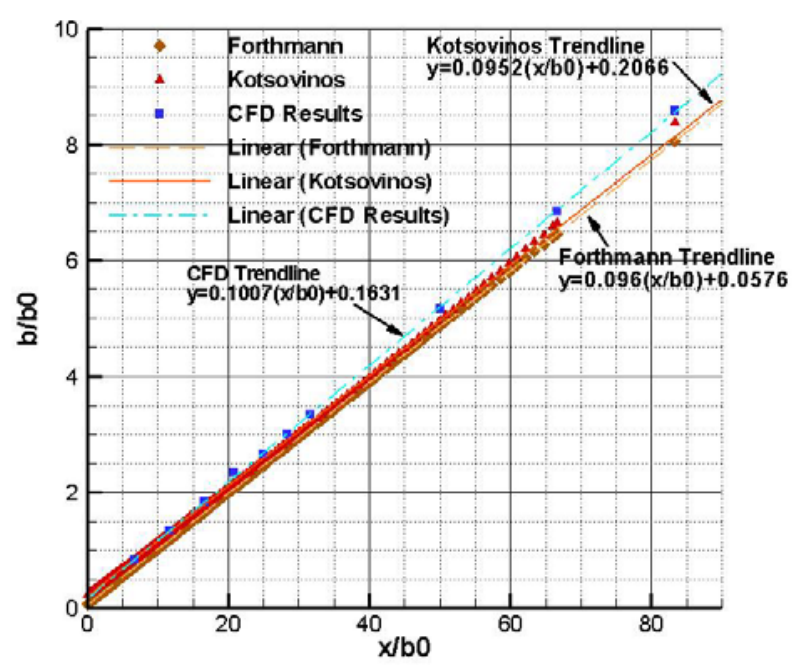

Fig. 4 - Angle of spread comparison above the centreline axis

Two important points can be drawn from equation (5):

- Firstly, $-\frac{K_{2}}{K_{1}}$ offers a glimpse of where the location of the virtual origin is when $b=0$. In this case, it is clear that the CFD virtual origin is located between Kotsovinos' and Forthmann's locations. Indeed, Kotsovinos' distance of the virtual point was found to be the farthest away, whereas Forthmann's is the closest to the real origin of the jet exhaust.

- Secondly, $\frac{K_{1}}{b_{0}}$ gives a measure of the spreading rate the jet is subject to. It can be seen that the CFD result shows a slightly higher rate of spreading than the other two curves. The difference is about $4.7 \%$ to Forthmann's experimental work and 5.5\% to Kotsovinos' empirical equation.

\subsubsection{Wall Jet}

A similar study as the one conducted for the free jet was also carried out for the wall jet. The jet is placed one diameter $\left(b_{0}\right)$ above the ground, and similar parameters were set up for the jet boundary conditions. The control volume had to be slightly modified to take the ground into account, and it was found that a control volume of $106.6 b_{0}$ by $110 b_{0}$ was sufficiently large to avoid the influences from the artificial boundaries. Following similar mesh-independence tests, the total mesh density considered for this control volume comprises 163,326 nodes.

The CFD results for the wall jet were compared with Forthmann's experimental results reported by Rajaratnam (1976), who only took into account data from $20 x / b_{0}$ behind the jet to show the vertical velocity pattern at different distances downstream. Fig. 5 shows that the general velocity profile is well predicted by the CFD simulation at different distances behind the jet. Slight differences are only found very near the wall and at the end of the shear layer. 


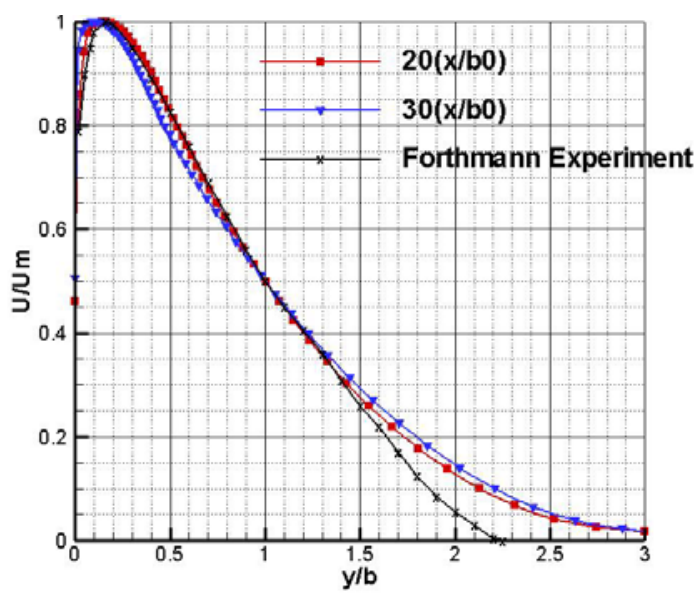

Fig. 5 - CFD and experimental results for the wall jet vertical velocity profile

The differences near the wall come from the fact that wall properties such as surface roughness are unknown in Forthmann's experiment (Tachie et al., 2004). The differences at the end of the shear layer may come from the fact that the experiment instruments were quite basic in Forthmann's experiment, which failed to comply with three criteria for assessing experimental data (Rodi, 1975). In addition, the results were taken very near the jet exhaust where the flow is supposed to exhibit considerable scatter at its edges, as explained earlier on.

Similar to the free jet study, it is possible to analyse the spreading parameters affecting the flow in the case of the wall jet. Fig. 6 shows a comparison between the spreading parameters for the free and wall jets.

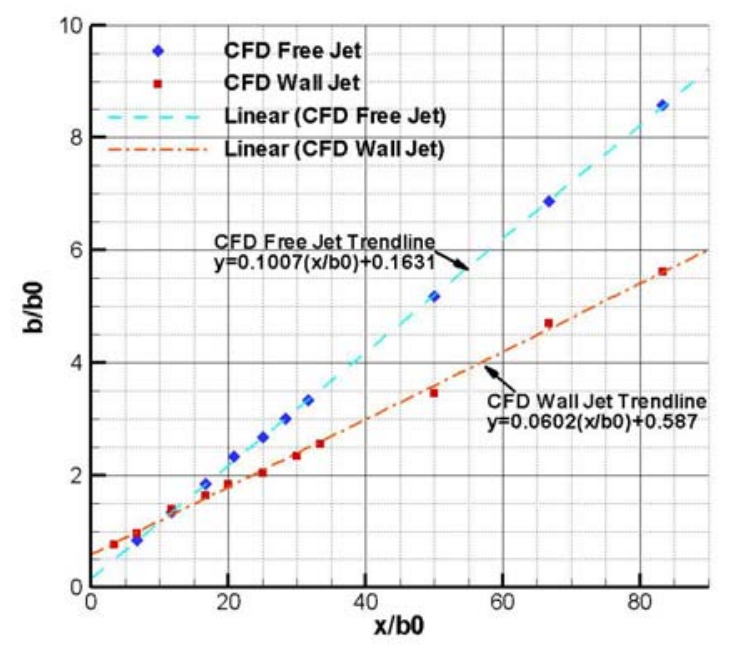

Fig. 6 - Angle of spread comparison between free and wall jets

Considering first the position of the virtual origin, its location is situated behind the actual origin of the wall jet and the value $-\frac{K_{2}}{K_{1}}$ gives a magnitude of $9.75 x / b_{0}$. This compares very well with the findings of Rajaratnam (1976), who predicted the location of the virtual origin to be around $10 x / b_{0}$ behind the nozzle.

Although the location of the virtual origin is accurately predicted by the CFD results, the angle of spread is slightly under-predicted with a difference of about $11.5 \%$ below the value given by Rajaratnam $(1976)(b=0.068 x)$. However, there are 
uncertainties regarding this parameter. Launder and Rodi (1983) found a growth rate in the order of $0.073 \pm 0.002$ but Tachie et al. (2004) found this value to be much higher (from 0.085 to 0.09 at low Reynolds numbers).

The position of the virtual origin is clearly different for the free and wall jets, with the wall jet located further behind the nozzle compared to the free jet. The spreading angle is more pronounced in the case of the free jet than for the wall jet. Launder and Rodi (1983) predicted this behaviour and showed that the growth rate of the free jet is more than 30\% above that of the wall jet. Similarly, Rajaratnam (1976) stated that the 'wall jet growth is about 0.7 times that of the free jet'. Tangemann and Gretler (2000) explained this behaviour by highlighting the wall's influence as the main reason for the reduction in the spreading growth in comparison to the free jet.

\subsection{Three-Dimensional Simulations}

Everitt and Robins (1978) showed that there are problems associated with HWA measurements of a jet issuing into still air. In addition, Ramaprian and Chandrasekhara (1985) and Namer and Otugen (1988) reported some other problems when temperature differences are introduced between the jet and the ambient fluid. These include "calibration drifts, sensitivity of the velocity sensors to temperature and vice-versa, and probe interference" (Ramaprian and Chandrasekhara, 1985). Everitt and Robins (1978) discussed one possible way of solving some of the problems by including an external parallel moving stream to the jet. They observed, like Rodi (1975), that this setup reduces the problem of high turbulent intensities in the outer regions of the flow and thus measurements errors. Both Wygnanski and Fiedler (1969) and Rodi (1975) argued that this type of flow is not self-preserving because of the co-flow surrounding it, but Everitt and Robins (1978) argued that the flow is approximately self-preserving and found that the jet issued in co-flow is similar to the one issuing in still air when "the excess velocity on the centreline is large with respect to the free stream velocity".

This section aims to compare the results of three-dimensional transient CFD simulations of co-flowing free and wall jets, both with buoyancy, to assess the effects of the presence of the wall on the fluid properties. The results will provide the first step of a staged approach towards representing the pollution dispersion of complete aircraft during the take-off phase, to provide dispersion modellers with a better understanding of the near-source dynamics of the flow. The take-off process is transient and herein represented by the full International Civil Aviation Organisation (ICAO) time-in-mode of 42 seconds characterising the take-off duration.

\subsubsection{Computational models}

Two different computational domains were used; a cylindrical shape for the free jet and a rectangular shape for the wall jet simulation, as shown in Fig. 7. The width and breadth of the computational domain were chosen so that there would be no interference between the flow and the boundary conditions on the sides of the control volume. In this respect, the external boundaries are defined as symmetry walls as represented in many similar CFD simulations, e.g. Wang et al (2006).

Different control volume sizes were tested in horizontal and vertical twodimensional trials and the geometries presented in Fig. 7 were selected for the complete three-dimensional simulations of the free and wall jets. After selection of the control volume geometry, initial mesh dependency tests were carried out both in the vertical and horizontal directions, similarly to the two-dimensional study, to investigate the effect of the mesh density on the dynamic subgrid scale model. The 
final mesh density for the free jet consists of $1,257,456$ nodes, while that for the wall jet has 3,965,760 nodes.

The jet exhaust is assigned right at the beginning of the control volume in accordance with the findings of Celik et al. (2006). Suto et al. (2004) provided a similar geometrical setup for their study on the free jet using classical Smagorinsky and dynamic Smagorinsky models. The inlet velocity is $80 \mathrm{~m} / \mathrm{s}$ with no swirl effects, and the jet releases hot gases $(690 \mathrm{~K})$ of NOx emitted at a mass fraction of $0.74 \times 10^{-4}$ from an exhaust diameter $b_{0}=0.93 \mathrm{~m}$, according to the parameters from Koutsourakis et al. (2006).

The faces adjacent to the exhaust are also defined as velocity inlets but with a magnitude $2.5 \mathrm{~m} / \mathrm{s}$ to replicate the co-flow condition of a headwind. The co-flowing wind is adiabatically stratified across the vertical direction. On the opposite side to the jet exhaust, an outflow boundary condition was used to model the flow exit.

The wall jet has an additional boundary condition; the bottom wall is set up as stationary with surface roughness of $0.003 \mathrm{~m}$, corresponding to the concrete PCN 60 R/B/W/T used at Zurich airport. Werner and Wengle's near-wall treatment (Fluent, 2005) was used to account for the boundary layer formation at the far field regions where the mesh is coarser. In this particular simulation, only the horizontal mesh spacing increases, while the vertical distribution remains the same throughout the control volume. The Werner and Wengle wall functions are based on the two-layer approximation of the viscous sub-layer taking into account the assumption that the one-seventh power law resides outside this layer, and provide a better representation of the near-wall layers (Farsimadan and Mokhtarzadeh-Dehghan, 2008).

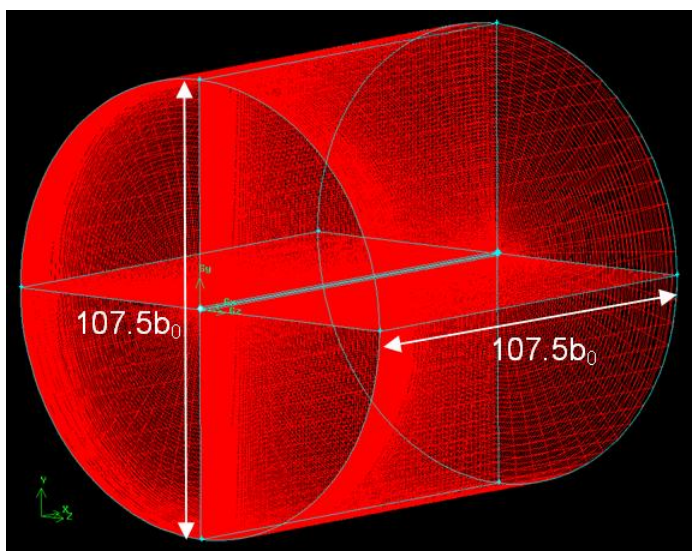

Free jet

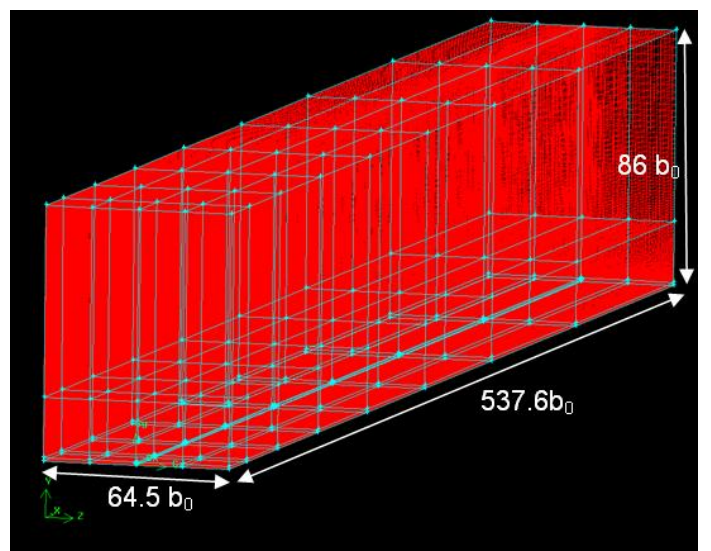

Wall jet

Fig. 7 - Geometry of the 3D free and wall jet domains

\subsubsection{Streamwise Direction}

An analysis of the results after $5 \mathrm{~s}$ shows that there is a slight difference in the length where the free and wall jets both reach $U_{m} /\left(U_{0}-2.5\right)=0$. The free jet attained this value faster than the wall jet; on the other hand, the potential core is much shorter and its decay rate is much greater for the free jet than for the wall jet. The results at 10s also show a similar behaviour upwind, but with a more elongated pattern because the fluid is still progressing through time and has not yet attained a steady-state condition (Fig. 8). 


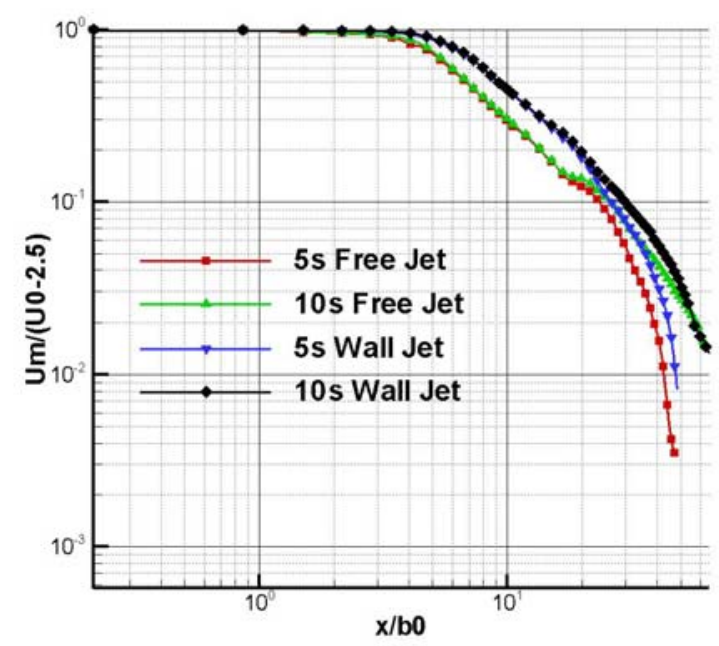

Fig. 8 - Maximum velocity decay comparison between free and wall jets

Fig. 9 shows a comparison of the mean velocity profile for the free jet (left) and the wall jet (right), at different times. The results after 1s show a much further penetration for the wall jet than for the free jet. The free jet still exhibits an almost symmetrical pattern, as in the case of a non-buoyant free jet. The wall jet, on the other hand, shows a different pattern with the fluid rising at some distance behind the jet exhaust.

Buoyancy effects can clearly be seen after $5 \mathrm{~s}$, when the free jet velocity profile shows a deviation from the centreline axis. The wall jet has approximately the same pattern as before, with the flow rising much higher than for the free jet. The flow penetration is also much deeper for the wall jet than for the free jet, as previously stated.

The results at time $=10$ s continue the previous trends, with greater flow penetration and higher rise for the buoyant wall jet than for the buoyant free jet. Another interesting point that can be observed for the wall jet is that, at large distances behind the exhaust, the flow separates from the wall. Although not so apparent, this effect can already be seen after 1s, at about 30 diameters behind the exhaust, and is clearly seen after 10s at about 64.5 diameters behind the exhaust.

The vertical velocity profile at different distances behind the exhaust for the buoyant wall jet is shown in Fig. 10. From the exit of the nozzle to a distance of $8 x / b_{0}$, the wall jet is still in the potential core and intermediate regions, whereas from this distance onwards the wall jet is in the fully developed region and exhibits a selfsimilarity profile.

Forthmann's experimental results are used to verify the self-similar profile. It can be seen that all the plots after $8 x / b_{0}$ behind the exhaust closely follow the experimental results, both in the boundary layer and the free shear layer.

A comparison between the rate of spread of the free and wall jets, at two different time levels, is given in Fig. 11. Both graphs show the same general pattern as for the $2 \mathrm{D}$ simulations presented earlier on. The rate of spread is greater for the free jet than for the wall jet. 

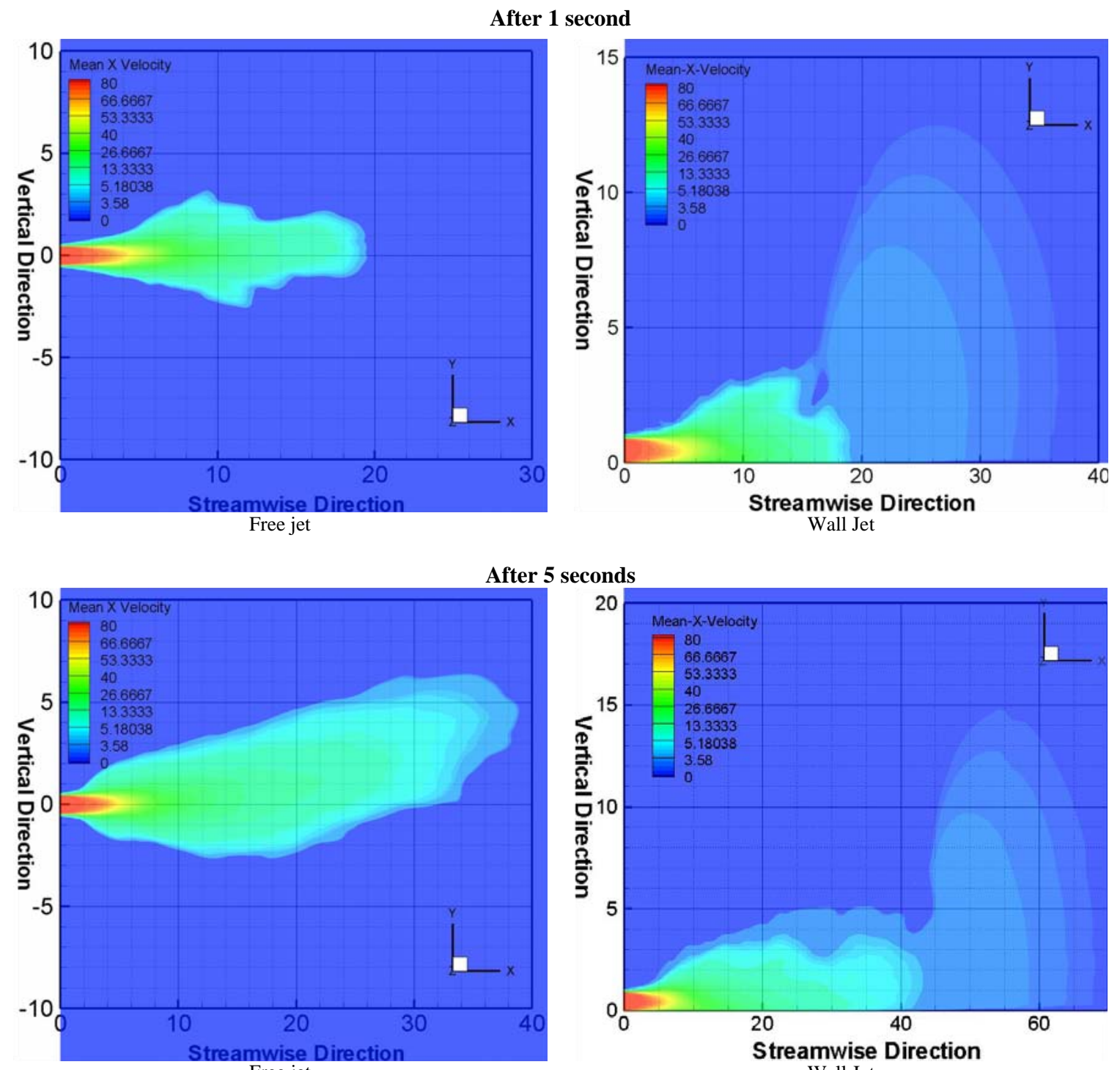

Free jet

After 10 seconds
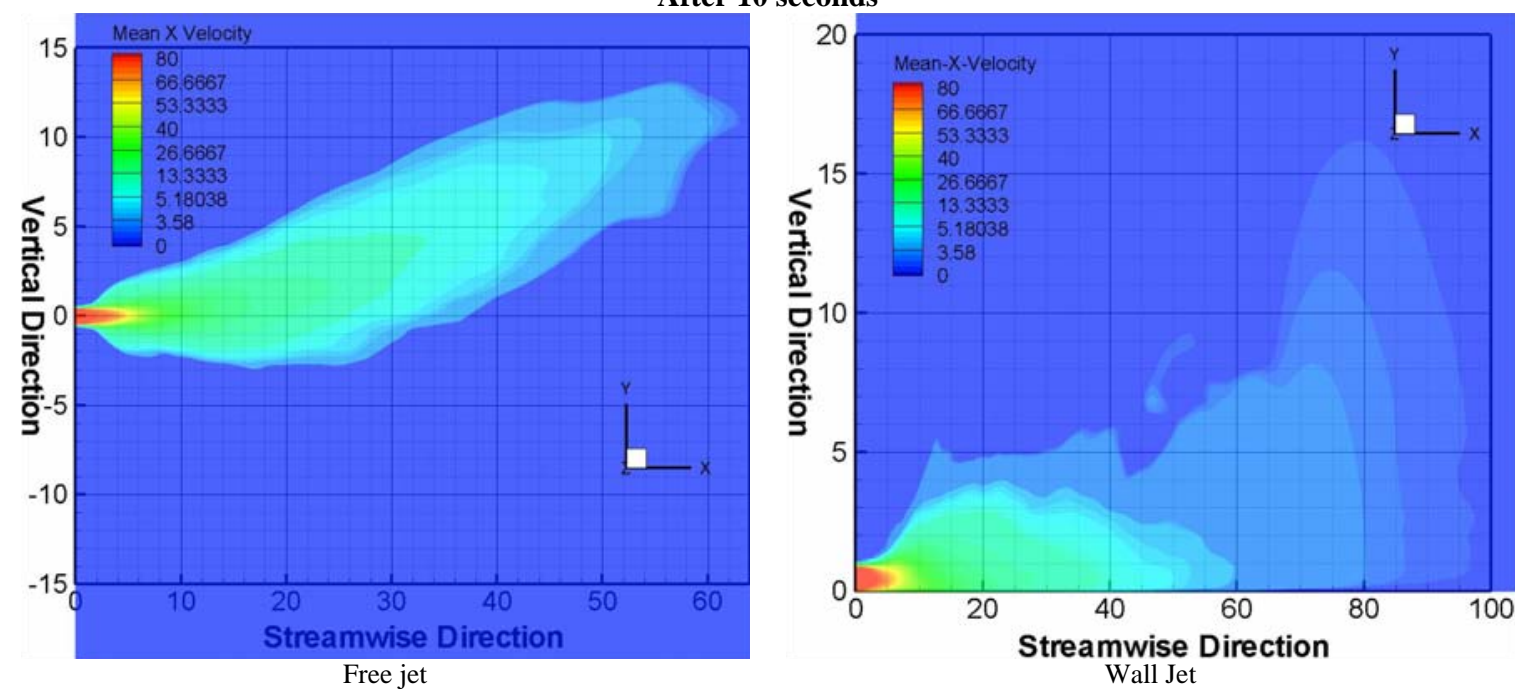

Fig. 9 - Mean velocity profile evolution through time for the buoyant free and wall jets 


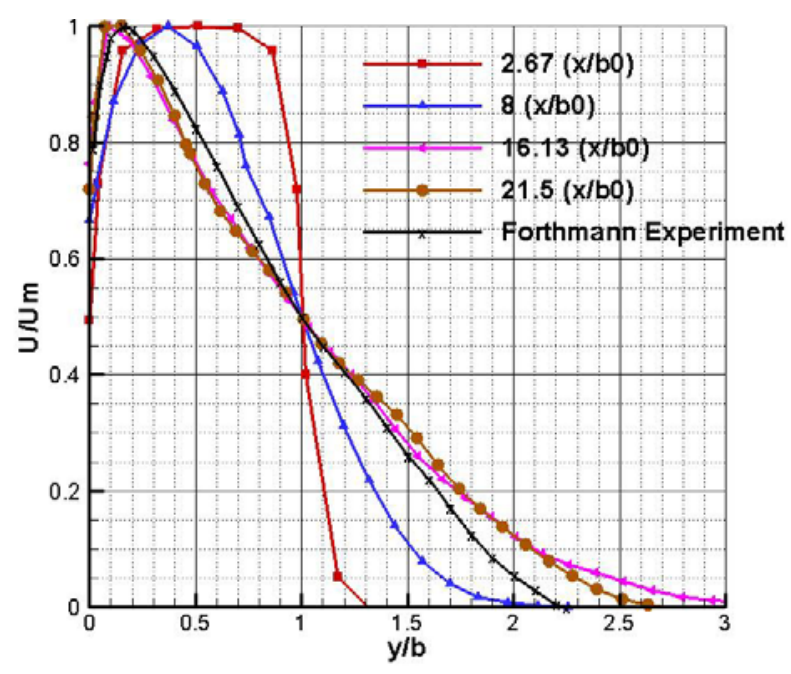

Fig. 10 - Streamwise velocity profiles at different distances behind the buoyant wall jet

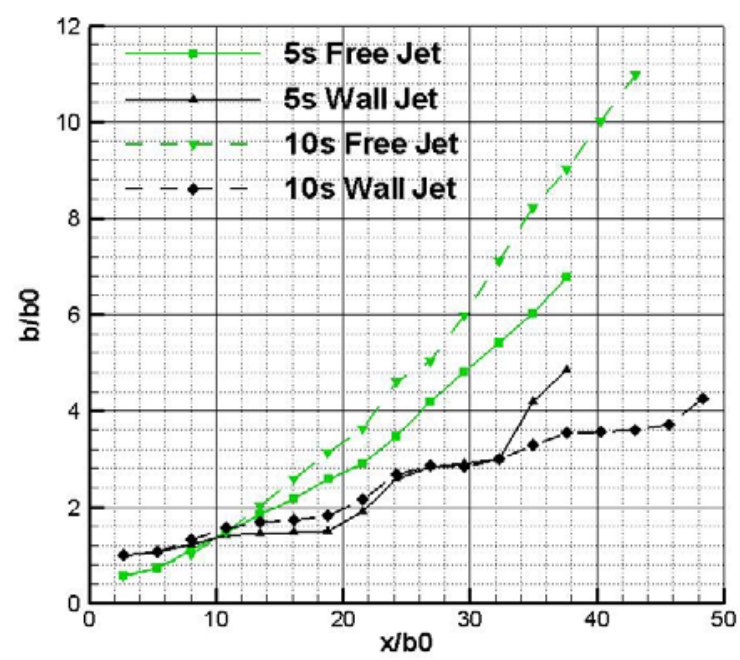

Fig. 11 - Angle of spread evolution of buoyant free and wall jets

As mentioned earlier, several authors e.g. Launder and Rodi (1983) had already observed this behaviour in a non-buoyant situation, and claimed that the growth rate of the free jet is more than $30 \%$ higher that of the wall jet. This trend tends to grow as the flow progresses through time. It is interesting to note that the growth rate is actually increasing with time for the buoyant free jet, whereas for the buoyant wall jet the growth rate is actually decreasing. This behaviour is due to the vortices generated by the presence of the wall; this is true for some distances behind the exhaust but, at larger distances, the wall effects decrease and the buoyancy effects may take over and lift the jet above the ground.

Fig. 12 gives a comparison of the height of the plume centreline as a function of downward distance. The ordinate axis is changed into the non-dimensional form $\left(y-b_{0}\right) / y_{m}$, with $y_{m}$ the maximum height of the wall jet simulation and $y$ defined as the distance where the maximum velocity is encountered downstream of the jet exhaust.

The rise above the actual centreline axis of the jet is much faster for the free jet than for the wall jet. The buoyant wall jet starts rising above the centreline at about $55 x / b_{0}$; before this point, the plot never reaches a positive value. It is either 
approaching the centreline value (0) or stays negative, meaning the flow is going down. This behaviour is also known as the clinging phenomenon due to the Coanda effect.

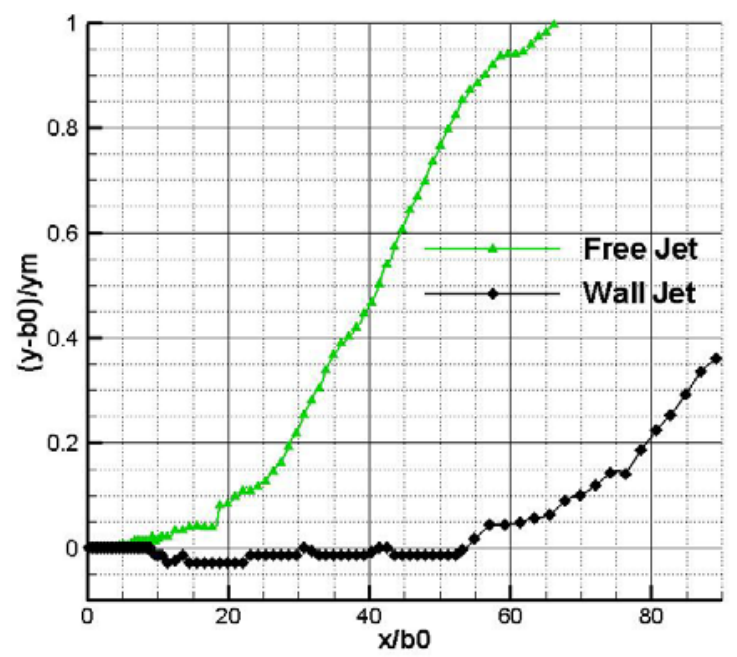

Fig. 12 - Comparison of the height of the plume centreline at different distances behind the exhaust, after 10s

Although in a steady-state condition, Sharp and Vyas (1977), analysing horizontal buoyant wall jets, found a crude linear relation between the relative distance $L / b_{0}$ over which the flow stays attached to the ground and the densimetric Froude number $\mathrm{Fr}$ :

$\frac{L}{b_{0}}=F r \times C$

with $c=3.2$ found experimentally and $L$ the length of cling. For this simulation, at the time when it reaches a steady-state condition, the length of cling should be $166 \mathrm{~m}$ behind the exhaust. At the time 10s, the length of cling in Fig. 12 is about $55 b_{0}=51.15 \mathrm{~m}$, which is in the region of the plume lift-off shown in Fig. 9.

A comprehensive literature review on the lift-off phenomenon was carried out by Ramsdale and Tickle (2001) in their study of ground-based buoyant-released clouds. One interesting parameter when studying the lift-off of a buoyant gas is the Richardson number $L_{p}$, given by:

$$
L_{p}=\frac{g H}{u_{*}^{2}} \times\left(\frac{\Delta \rho}{\rho_{a}}\right)
$$

with $H$ the effective depth, $u_{*}$ the friction velocity, $g$ the gravitational acceleration and $\Delta \rho$ the difference in density between the ambient fluid and the jet.

Briggs (Ramsdale and Tickle, 2001) was the first to propose an approximate value for $L_{p}$ in the order of 2.5 to 10, but he recognized himself that these values are not entirely correct. Hanna et al. (1998) reviewed wind tunnel studies and concluded that this value should be raised to about 20, with an uncertainty of a factor of two. Even these experimental results are not very reliable as the technique used to obtain 
the data was very basic, as Meroney (1979) relied on the average optical judgement of observers whereas Sinclair et al. (1990) found this distance by 'linear interpolation between the neighbouring vertical cross sections of their measured temperature field'.

Sinclair et al. (1990) collected some of the parameters that play an important role in the plume lift-off; these include the jet temperature, the jet exhaust velocity and the jet orifice geometry. They agreed with Ramsdale and Tickle (2002) on the influence of the jet to ambient velocity ratio and the jet geometry aspect ratio on the plume lift-off. All else being equal, a higher jet to ambient velocity with a low aspect ratio increases the clinging length, and hence delays the lift-off, whereas a higher aspect ratio shortens the lift-off from the ground.

\subsubsection{Spanwise Direction}

The symmetrical properties of the flow in the spanwise direction can be seen in Fig. 13. The velocity profiles at different distances behind the jet are compared with theoretical curves of Goertler (Abramovich, 1963), Tollmien (Abramovich, 1963) and Bradbury (1965).

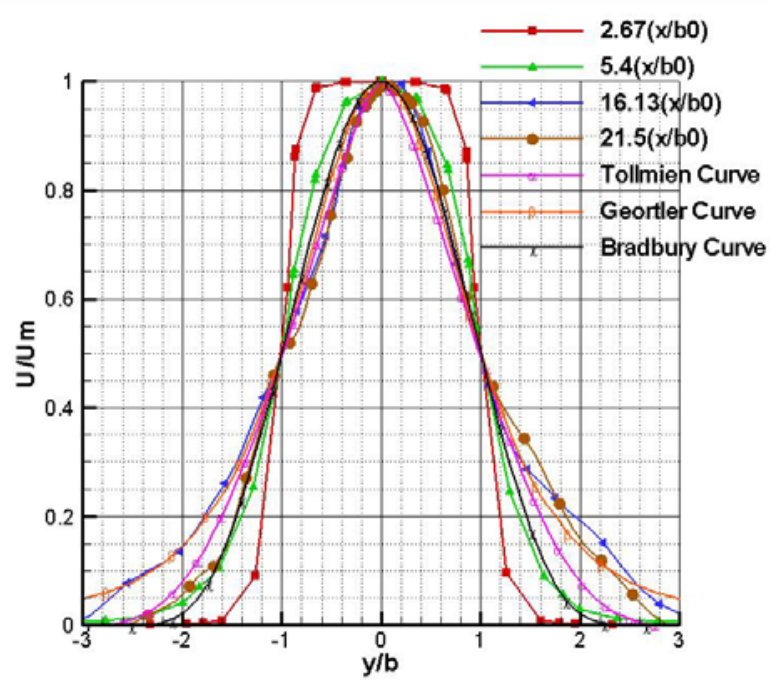

Fig. 13 - Spanwise velocity profile comparison of wall jet with theoretical curves of the free jet after 10s

The wall jet results show a profile similar to the free jet reported earlier on. The characteristic Gaussian-like distribution of the free jet simulation can be seen in this figure. The curves are different for the first two distances behind the exhaust $\left(2.47 x / b_{0}\right.$ and $\left.5.4 x / b_{0}\right)$ because the jets are still in the potential core and the flow development region.

All the plots are very much alike after $5.4 x / b_{0}$, showing a self-similar pattern. A comparison between the theoretical and CFD results reveals that, in the inner region, they are very much similar but some differences occur in the outer layer. Comparable to the results of the free jet simulation, these were attributed to the mixing with the ambient surrounding occurring in this region.

Fig. 14 shows a comparison of the rate of spreading between the free and wall jet simulations. Even though upstream the rate of spread seems to be higher for the free jet, further downwind this situation is reversed and the wall jet grows more rapidly. 
Another comparison can be made concerning the wall jet simulation with the one presented in Fig. 11. The vertical spread of the wall jet is very much smaller than the spanwise spread. This observation was also made by several authors, such as Rajaratnam (1976) and Swamy and Bandyopadyay (1975). They all found that the growth rate of the wall jet in the spanwise direction is much larger than in the vertical direction. This difference in rate of spread leads to the formation of two distinctive virtual origins. In the vertical direction presented in Fig. 11, the virtual origin can be clearly seen to be situated upstream of the actual position of the jet exhaust. But in the spanwise direction in Fig. 14, if the graph had started at $8 x / b_{0}$ (lower than this distance corresponds to the potential core and flow development region), the virtual origin would have been situated this time far downstream of the actual position of the jet exhaust. This discussion confirms the findings of Swamy and Bandyopadyay (1975), who found this wide separation to be about $12.5 \times / b_{0}$ between the two virtual origins; the vertical one being situated "upstream of the orifice" and the spanwise one located "downstream of the orifice".

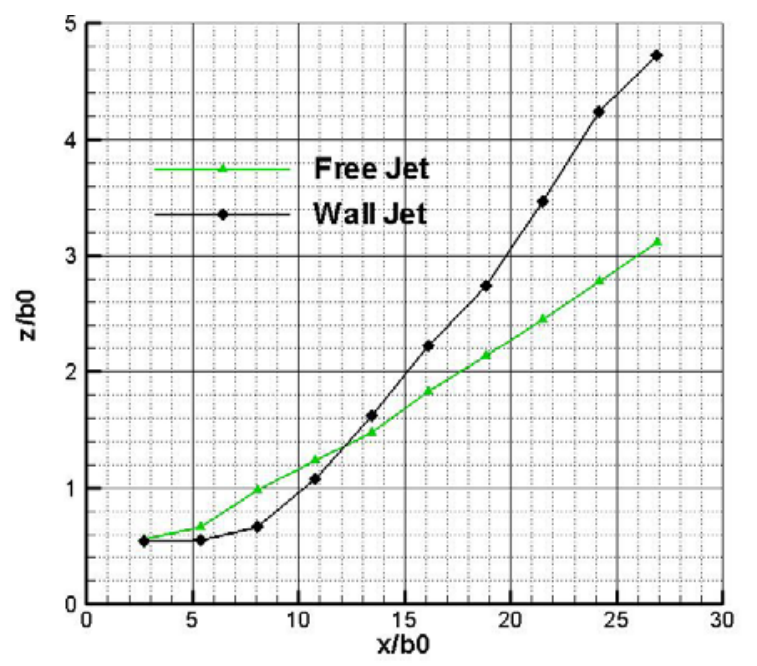

Fig. 14 - Spanwise direction rate of spread comparison after 10s

\subsubsection{Vorticity Profile Comparison}

In order to better understand the role vortices play in the dispersion process of buoyant free and wall jets, contours of instantaneous velocity profile were taken with similar scale and the addition of an iso-surface of $80 \mathrm{~m} / \mathrm{s}$ included with its grid represented in black.

The roll-up of the shear layers around the jet can be seen in Fig. 15, which plots velocity iso-surfaces. This is closely linked to the vorticity parameter in the sense that the roll-up pattern is created by the entrained eddies breaking the fluid. These entrainment vortices are situated in the local areas where the jet velocity is lower than the surrounding velocity. Rajaratnam (1976) also found that the disappearance of the potential core is a result of turbulence penetration, leading to the fully developed region. There is a close relationship between the velocity and vorticity parameters as will be shown later. 


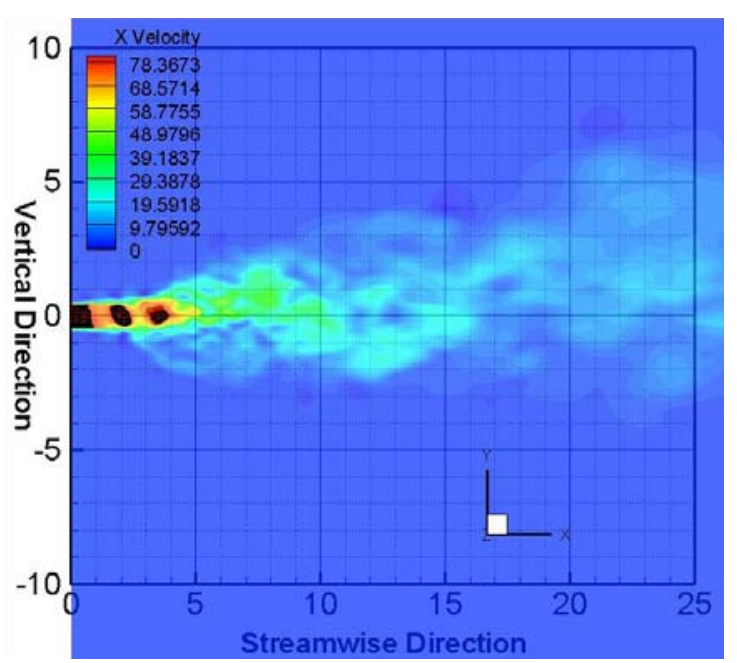

Buoyant free jet

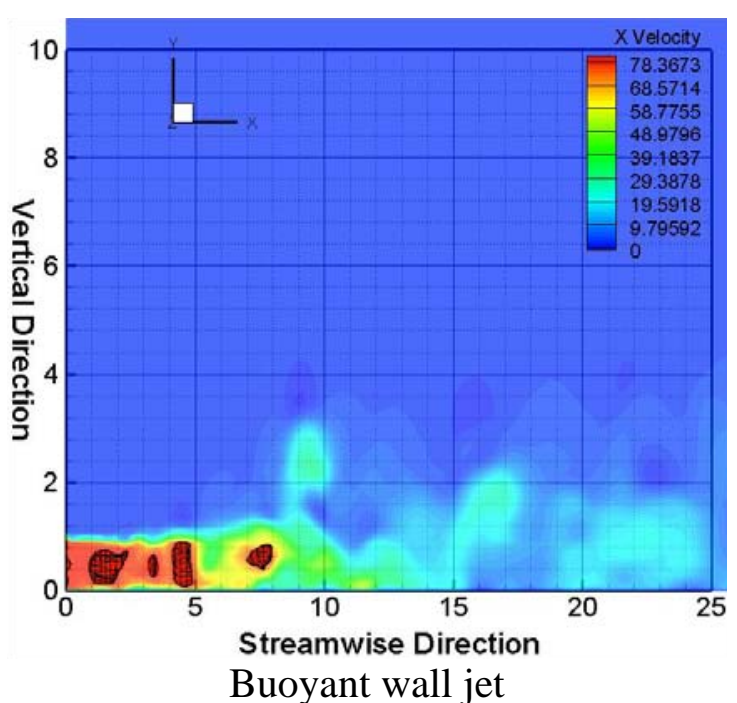

Buoyant wall jet

Fig. 15 - Velocity profile comparison at 5s

There are several other points that can be drawn from Fig. 15. Firstly, the potential core is much longer for the buoyant wall jet than for the buoyant free jet. This statement can be made by analysing the velocity iso-surface. While in the wall jet simulation there is one iso-surface extending to about $8 \mathrm{~m}$ behind the exhaust, the free jet shows its last iso-surface to be at a distance less than $5 \mathrm{~m}$. The wall jet potential core length behind the exhaust is deeper than a similar free jet. This point can also be observed in Fig. 16, where the point of contact between the upper and bottom vorticity contours marks the end of the potential core length. As can be seen, the point of merging is much further for the wall jet than for the free jet simulation.

Finally, Fig. 15 gives an insight on the spreading rate. At large distances behind the exhaust, the velocity contours go much higher for the free jet than its wall jet counterpart. At about $20 \mathrm{~m}$ behind the jet exhaust, the velocity contours reach $4 \mathrm{~m}$ in the positive vertical direction for the free jet but only $2 \mathrm{~m}$ for the wall jet. This statement is also in line with the discussion on the rate of spreading, and confirms that the growth rate is higher for the free jet than the wall jet. As mentioned previously, this observation can also be made from the analysis of vorticity contour plots, where contours of vortices for the wall jet rise at far greater distances than for the free jet simulation.

The roll-up process was earlier attributed to the entrainment eddies located outside the jet domain. Fig. 16 presents the magnitude of the vorticity vector defined as $\sqrt{(X \text { Vort })^{2}+(\text { YVort })^{2}+(Z \text { Vort })^{2}}$ and clearly shows this, with vortices of higher magnitude concentrated near the jet radius and the wall for the wall jet simulation. The magnitude of the vortices is much higher for the wall jet than for the free jet simulation, especially near the jet exhaust. It can also be seen that the vortices are more numerous for the free jet than for the wall jet simulation. This observation was also made by Townsend (1976), who attributed this behaviour to the presence of a solid boundary. 


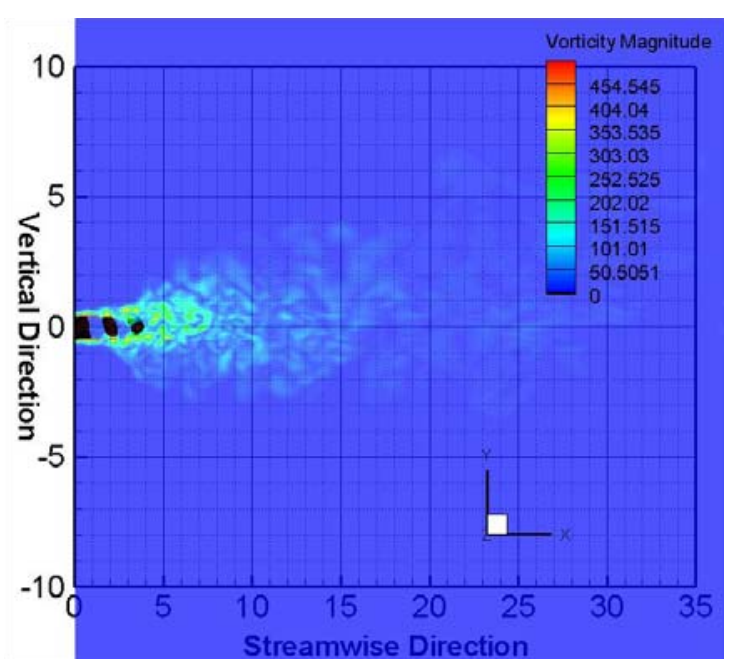

Buoyant free jet

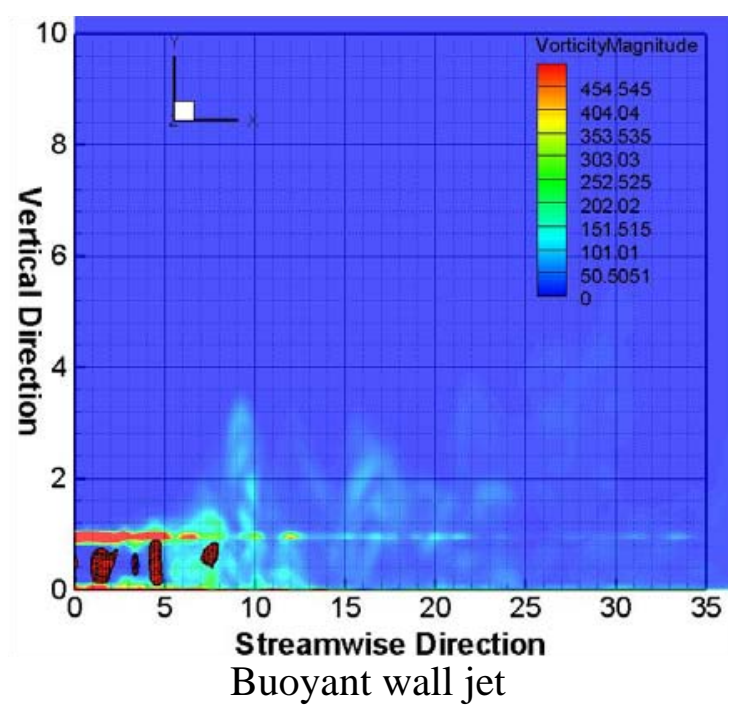

Fig. 16 - Vorticity magnitude comparison at $5 s$

One of the main characteristics of a jet with or without a solid boundary concerns the spreading in the spanwise direction caused by the streamwise vortices. Liepmann (1995) showed that the formation of strong streamwise vortical structures increases the entrainment rate of an obstructed jet as compared to a free jet, and this is the cause of the faster growth in the spanwise direction dispersion as shown earlier in the paper. According to Gholamreza-Kashi et al. (2004), the higher growth rate in the spanwise direction happened at the "cost of the growth of the jet" in the streamwise direction.

The rotation around the z-axis, also known as the spanwise vorticity, was found to be the most interesting for this study. It can be seen from Fig. 17 that counter-rotating vortices are formed right at the jet exhaust. The positive vortices are caused by the shearing mechanism of the jet flow encountering the moving surrounding fluid but, unlike the free jet simulation, the negative vortices present at the bottom are due to the wall.

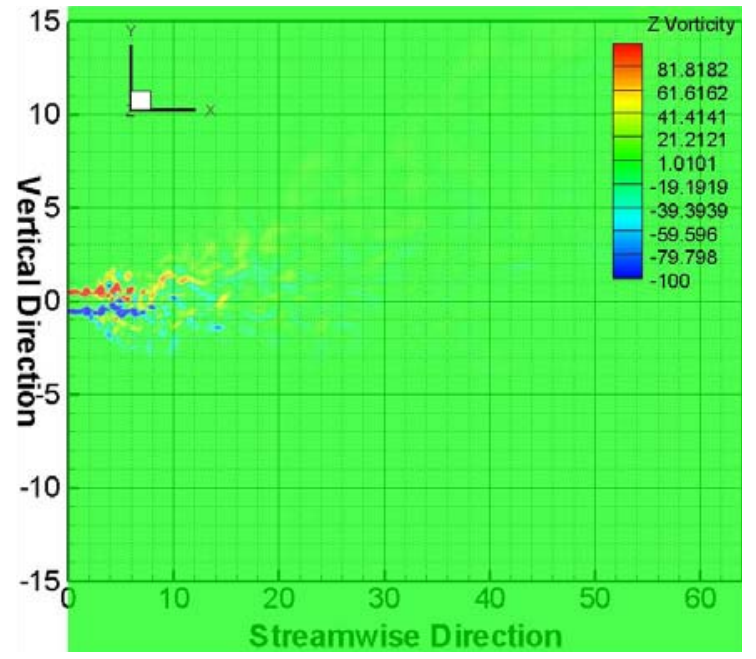

Buoyant free jet

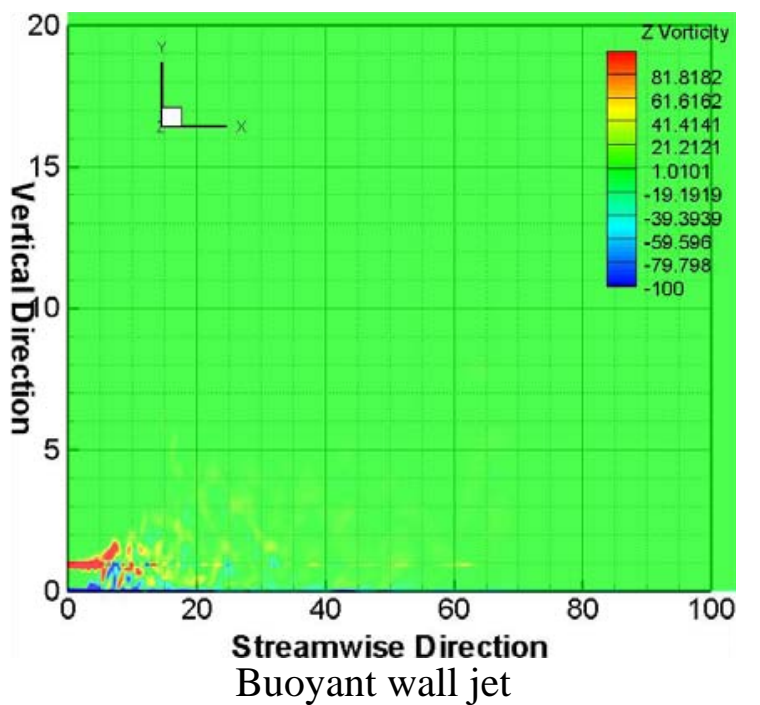

Buoyant wall jet

Fig. 17 - Spanwise vorticity comparison between buoyant free and wall jets at 10s

The merge between the positive and negative vortices happens further away from the jet exhaust for the wall jet than for the free jet. This merge corresponds to the disappearance of the potential core and, from this comparison, it can be seen that the 
potential core is much longer for the wall jet than the free jet. As previously mentioned, this has an effect on the penetration through the control volume and the rate of decay of the maximum velocity. The wall jet simulation shows a deeper penetration and lower decay rate through the control volume.

As can be seen in Fig. 17, the number of vortices is lower in the wall jet simulation than the free jet simulation, confirming the statement made by Townsend (1976). Both simulations show that the vortices first undergo a coalescence cascade before breaking up, the only difference between them being that the influence of the negative vortices created by the solid boundary still remains important. This has an effect on the flow pattern in terms of keeping it down. As illustrated previously, the negative vortices force the flow to cling to the wall.

After the clinging length, the flow was found to lift-off from the ground with large gusts as found by Sharp and Vyas (1977). Sinclair et al. (1990) discussed the importance of the spanwise vortices in the role they play in lifting-off the wall jet from its solid boundary.

\section{Conclusions}

The current study represents the first step towards a better understanding and proper representation of the source dynamics behind an airplane jet engine during the takeoff and landing phases. This study provides a comparison between free and wall jets, by first validating the CFD simulations with quantitative results from previous analytical and experimental works. Then, it offers an in-depth discussion of the flow physics generated by buoyant free and wall jets in similar conditions as the engine of an aircraft during taking-off.

The near-field flow properties of turbulent buoyant free and wall jets in a coflowing situation was discussed in this paper by means of LES simulations. The comparison between the buoyant free and wall jets revealed several differences: first, the potential core region is much longer for the wall jet than for the free jet. This has an effect on the flow penetration through the control volume; the wall jet offers a deeper penetration than the free jet case. A correlation can be found between this parameter and the maximum velocity decay as the penetration involves higher velocity pushing into the control volume. The maximum velocity decay is much faster for the free jet than for the wall jet. Finally, the spreading rate was found to be higher in the case of the buoyant free jet.

All the parameters discussed above are interconnected with the spanwise vortical structure of the buoyant wall jet. As in the case of the buoyant free jet, counter-rotating vortices are created on one side by the surrounding fluid and on the other by the solid boundary. What is different from the free jet situation is the presence of the wall generating vortices throughout the control volume, while the influence of the vortices created by the surrounding fluid gradually decreases.

The first point of merging of the counter-rotating vortices occurs in the potential core length. As the flow progresses, the intensity of the vortices created by the wall are much stronger than the ones created by the surrounding fluid, causing the flow to cling to the wall. This pushing-down phenomenon restricts the growth of the jet, hence a lower rate of spread than for the buoyant free jet. As the velocity further away from the jet exhaust decreases, the vortices created by the wall decrease and buoyancy takes over, with positive vortices lifting up the flow from the ground.

The information generated in this paper provides an initial understanding of the plume dynamics created by an aircraft engine in the take-off phase. Future steps will be taken by raising the jet from the ground and including different parts of an 
airplane. The results of this final step will be compared with Light Detection and Ranging (LIDAR) measurements of an aircraft made at Manchester airport. Other studies of high relevance in the context of an airport dispersion model will be initiated such as including non-linear chemistry to shed light on the chemical transformations of different species.

\section{Acknowledgment}

The work reported in this paper has been undertaken as part of the Airport Local Air Quality Studies (ALAQS) project commissioned and sponsored by EUROCONTROL. The authors would like to thank all the members of ALAQS consortium, especially Dr. Ian Fuller from EUROCONTROL. The authors would also like to thank the anonymous reviewers who gave valuable comments and suggestions for the improvement of this paper. 


\section{References}

Abramovich, G.N., 1963. The Theory of Turbulent Jets. MIT Press.

Aloysius, S. S., Pearce, D., Wrobel, L.C. and Silue, M., 2006. Comparison of CFD with Lagrangian-based Simulations for Airfield Emissions Dispersion. Report, EUROCONTROL Experimental Centre.

Archer, R.D. and Saalas, M., 1996. An Introduction to Aerospace Propulsion. Prentice Hall.

Aziz, T.N., Raiford, J.P. and Khan, A.A., 2008. Numerical simulation of turbulent jets. Engineering Applications of Computational Fluid Mechanics 2, 234-243.

Bakker, A., Modelling of Turbulence in Stirred Vessels using Large Eddy Simulation, 2004. FLUENT online CFD Book.

Bradbury, L.J.S, 1965. The structure of a self-preserving turbulent jet. Journal of Fluid Mechanics 23, 31-64.

Celik, I., Klein, M., Frietag, M. and Janicka, J., 2006. Assessment measures for URANS/DES/LES: An overview with applications. Journal of Turbulence 7, 1-27.

Celikel, A., Duchene, N., Fuller, I., Silue, M., Fleuti, E., Hofmann, P. and Moore, T., 2004. Airport Local Air Qualities, Case Study: Emission Inventory for Zurich Airport with Different Methodologies. Report EEC/SEE/2004/010, EUROCONTROL Experimental Centre.

Deo, R.C., Mi, J. and Nathan, G.J., 2007. The influence of nozzle-exit geometric profile on statistical properties of a turbulent plane jet. Experimental Thermal and Fluid Science 32, 545-559.

Everitt, K.W. and Robins, A.G., 1978. The development and structure of turbulent plane jets. Journal of Fluid Mechanics 88, part 3, 563-583.

Farsimadan, E. and Mokhtarzadeh-Dehghan, M.R., 2008. A large eddy simulation of an airfoil turbulent wake subjected to streamwise curvature. International Journal for Numerical Methods in Fluids 56, 1233-1240.

FLUENT Inc. Product documentation, 2005.

Forthmann, E., 1936. Turbulent Jet Expansion. English Translation, NACA Technical Memorandum TM-789.

Gholamreza-Kashi, S., Martinuzzi, R.J. and Baddour, R.E., 2004. Turbulence measurements in a rectangular surface jet. $17^{\text {th }}$ ASCE Engineering Mechanics Conference, 13-16 June 2004, Newark, DE. 
Hanna, S.R., Briggs, G.A. and Chang, J.C., 1998. Lift-off of ground-based buoyant plumes. Journal of Hazardous Materials 59, 123-130.

Haworth, D.C. and Pope, S.B., 1987. A pdf modelling study of self-similar turbulent free shear flows. Physics of fluids 30 (4), 1026-1044

Kotsovinos, N.E., 1976. A note on the spreading rate and virtual origin of a plane turbulent jet. Journal of Fluid Mechanics 77, 305-311.

Koutsourakis, N., Bartzis, J.G., Venetsanos, A. and Rafaildis, S., 2006, Computation of pollutant dispersion during an airplane take-off. Environmental Modelling and Software 21, 486-493.

Launder, B.E. and Rodi, W., 1983. The turbulent wall jet - Measurements and modelling. Annual Review of Fluid Mechanics 15, 429-459.

Liepmann, D., (1995). Why do streamwise vortices form at the top and bottom of a round jet moving parallel to a free surface?. Journal of Fluid Engineering ASME 117, 205-206.

List, E.J., 1982. Turbulent jets and plumes. Annual Review of Fluid Mechanics 14, 189-212.

Meroney, R.N, 1979. Lift-off of buoyant gas initially on the ground. Journal of Industrial Aerodynamics 5, 1-11.

Mi, J., Deo, R.C. and Nathan, G.J., 2005. Characterisation of turbulent jets from highaspect-ratio rectangular nozzles. Physics of Fluids 17, 068102.

Mi, J., Kalt, P., Nathan, G.J. and Wong, C.Y., 2007. PIV measurements of a turbulent jet issuing from round sharped-edged plate. Experiments in Fluids 42, 625-637.

Mi, J., Nathan, G.J, and Luxton, R.E., 2000. Centreline mixing characteristics of jets from nine differently shaped nozzles. Experiments in Fluids 28, 93-94.

Morchain, J., Maranges, C. and Fonade, C., 2000. CFD modelling of a two-phase jet aerator under influence of a crossflow. Water Research 34, 3460-3472.

Namer, I. and Otugen, M.V., 1988. Velocity measurements in a plane turbulent air jet at moderate Reynolds numbers. Experiments in Fluids 6, 387-399.

Penner, J.E, Lister, D.H., Griggs, D.J., Dokken, D.J. and McFarland, M., 1999. Aviation and the Global Atmosphere, http://www.grida.no/climate/ipcc/index.htm.

Rajaratnam, N., 1976. Turbulent Jets. Series in Developments in Water Science, Volume 5, Elsevier.

Ramaprian, B.R. and Chandrasekhara, M.S., 1985. LDA measurements in plane turbulent jets. Journal of Fluid Engineering ASME 107, 264-271. 
Ramsdale, S.A. and Tickle, G.A., 2001. Review of Lift-off Models for Ground Based Buoyant Clouds. AEA Technology Report AEAT-4262 (2) for EC URAHFREP Project.

Ribeiro, M.M. and Whitelaw, J.H., 1980. The structure of turbulent jets. Proceedings of the Royal Society of London A 370, 281-301.

Rodi, W., 1975. A Review of Experimental Data of Uniform Density Free Turbulent Boundary Layers. Studies in Convection: Theory, Measurement and Applications, Volume 1, Academic Press.

Sharp, J.J. and Vyas, B.D., 1977. The buoyant wall jet. Proceedings of the Institution of Civil Engineers 63, Part 2, 593-611.

Sinclair, J.R., Slawson, P.R. and Davidson, G.A., 1990. Three-dimensional buoyant wall jets released into a coflowing turbulent boundary layer. Journal of Heat Transfer 112, 356-362.

Suto, H., Matsubara, K., Kobayashi, M. and Kaneko, Y., 2004. Large eddy simulation of flow and scalar transport in a round jet. Heat Transfer-Asian Research 33 (3), 175-188.

Swamy, N.V.C. and Bandyopadyay, P., 1975. Mean and turbulence characteristics of three-dimensional wall jets. Journal of Fluid Mechanics 71, 541-562.

Tachie, M.F., Balachandar, R. and Berstom, D.J., 2004. Roughness effects on turbulent plane wall jets in open channel. Experiments in Fluids 37, 281-292.

Tangemann, R. and Gretler, W., 2000. Numerical simulation of a two-dimensional turbulent wall jet in an external stream. Forschung im Engenieurwesen 66, 31-39.

Thorne, K., 2004. Application of classical physics - Chapter 14: Turbulence. Caltech Lecture Course Ph136. http://www.pma.caltech.edu/Courses/ph136/yr2004/.

Townsend, A.A., 1976. The Structure of Turbulent Shear Flow. Cambridge Monographs on Mechanics and Applied Mathematics, Cambridge University Press.

Van der Hegge Zijnen, B.G., 1958. Measurements of the Velocity Distribution in a Plane Turbulent Jet of Air. Applied Scientific Research 7, 256-276.

Wang. J.S., Chan, T.L., Cheung, C.S., Leung, C.W. and Hung, W.T., 2006. Threedimensional pollutant concentration dispersion of a vehicular exhaust plume in the real atmosphere. Atmospheric Environment 40, 484-497.

Wygnanski, I. and Fiedler, H., 1969. Some measurements in the self-preserving jet. Journal of Fluid Mechanics 38, 577-612. 